\title{
The utility of HACOR score in predicting failure of high-flow nasal oxygen in acute hypoxemic respiratory failure
}

\begin{abstract}
Objectives: To assess the diagnostic performance of HACOR scoring system using bedside variables and to predict failure of HFNO in patients with acute hypoxemic respiratory failure (AHRF).

Material and methods: 150 patients with AHRF who were receiving HFNO were enrolled in this study; to predict HFNO treatment failure. A scoring scale (HACOR score) consisted of Heart rate (beats/minute), acidosis (assessed by pH), consciousness (assessed by Glasgow coma score), oxygenation, and respiratory rate. Failure was defined as the need for intubation or death. Results: Patients were analyzed according to the success or failure of HFNO. Total 150 patients, of which $100(66.7 \%)$ had a successful treatment while $50\left(33.3 \%\right.$ ) failed with such intervention. There was an improvement in $\mathrm{HR}$ and $\mathrm{RR}$, and $\mathrm{PaO}_{2} / \mathrm{FiO}_{2}$ within the first hour (T1) in the success group and these parameters continued to improve even after 24 hours (T2) of HFNO treatment. Patients with HFNO failure had a higher HACOR score at initiation and after 1, 12, 24 and 48 hours. Before intubation, the highest value of the HACOR score was reached in the failure group. At $1 \mathrm{~h}$ of HFNO assessment, the area under the receiver operating characteristic curve was 0.86 , showing good predictive power for failure. We found that HACOR score at a cutoff point $>6$ had $81.2 \%$ sensitivity and $91 \%$ specificity, $92.5 \%$ positive predictive value, and $71.4 \%$ negative predictive value with a diagnostic accuracy was $85 \%$. Furthermore, the overall diagnostic accuracy exceeded $87 \%$ when the HACOR score was assessed at 1,12 , 24 or $48 \mathrm{~h}$ of HFNO.
\end{abstract}

Conclusions: The HACOR scale is a clinically useful bedside tool for the prediction of HFNO failure in hypoxemic patients. A HACOR score $<6$ after 1 hour of HFNO highlights patients with $<85 \%$ risk of failure.

Key words: hypoxemic respiratory failure, critical care

Adv Respir Med. 2021; 89: 23-29

\section{Introduction}

High-flow nasal oxygen (HFNO) is an innovative system that allows for delivering a high flow of heated and humidified gas up to $60 \mathrm{~L} / \mathrm{min}^{-1}$ and $0.21-1.0$ of $\mathrm{FiO}_{2}$ through a special nasal cannula [1].

HFNO has been increasingly conducted to treat acute hypoxemic respiratory failure (AHRF) patients [2]. Recent studies have compared the efficacy and outcome of HFNO with conventional oxygen therapy in (ICU) settings; indicate that HFNO demonstrates beneficial effects in terms of better oxygenation, as well as reduction of respiratory rate and dyspnoea, resulting in improving patient comfort $[2,3]$.
Using of HFNO can avoid intubation in patients with respiratory failure by temporarily supporting ventilation during initial treatment, but many subjects failed and ultimately need intubation. Patients at risk for HFNO failure may benefit from early intubation or close observation. There is a limited prediction tool to help clinicians to determine clinical outcomes and success rate in patients treated with HFNO.

Duan et al. have derived and validated a scoring system which accurately predicts patients that would be at risk of noninvasive (NIV) failure such that the clinician can plan for the decision to implement invasive mechanical ventilation. In a derivation cohort of 449 patients, the authors used stepwise multivariable regression analysis

Address for correspondence: Doaa M Magdy, Department of Chest Diseases, Faculty of Medicine, Assuit University, Egypt; e-mail: doaamagdy_2020@aun.edu.eg DOI: 10.5603/ARM.a2021.0031

Received: 07.09.2020

Copyright (C) 2021 PTChP

ISSN 2451-4934 
to identify variables predicting NIV failure. Each of the five parameters identified - heart rate, acidosis, consciousness (defined by the Glasgow Coma Scale [GCS] score), Oxygenation, and Respiratory rate (HACOR) - was assigned points, that added together to give an overall HACOR score. Hypothesizing that, the combination of these bedside variables has the potential to increase the predictive power for the prediction of NIV failure [4].

Hence, this study aims to assess a bedside scoring system based on five variables easily assessed in the emergency room (the HACOR score: heart rate, acidosis, consciousness, oxygenation, respiratory rate), to predict failure risk in patients with hypoxemic ARF treated with HFNO, the need for intubation. Hence the clinician can plan for the decision to implement invasive mechanical ventilation.

\section{Material and methods}

We conducted an observational prospective study in a 30-bed respiratory ICU at Assuit University Hospital between January 2018 and February 2020. This study was approved by the Faculty of Medicine Ethics Committee, Assiut University.

All the consecutive patients fulfilling the diagnostic criteria of acute hypoxic respiratory failure (AHRF) admitted to the ICU [5], and treated with HFNO were included after taking proper consent.

The inclusion criteria were age $>18$ years, presence of clinical signs and symptoms of AHRF; defined by 1) recent dyspnea with a breathing frequency $>25$ breaths/min and/or use of accessory muscles of respiration with pulmonary infiltrates on chest X-ray; 2) a $\mathrm{PaO}_{2} / \mathrm{FIO}_{2}$ of $>300 \mathrm{~mm} \mathrm{Hg}$ recorded during spontaneous oxygen ventilation at $15 \mathrm{~L} / \mathrm{min}^{-1}[5]$.

Patients who had an underlying chronic respiratory disease, who require emergent endotracheal intubation, Inability to protect airway (excess secretions, drowsy, or comatose patient), severe hemodynamic instability (patient on inotropic or vasopressor support), uncooperative patient, facial trauma or burns, facial surgery, or facial anatomical abnormality) were excluded [6].The demographic data of patients such as age, sex, the aetiology of acute respiratory failure, and presence of associated comorbid illnesses were recorded. The disease severity was calculated using the Acute Physiology and Chronic Health Evaluation II (APACHE II) score on admission to ICU [7].
Variables for HACOR score including; [heart rate (HR), respiratory rate (RR), consciousness (Glasgow Coma Scale (GCS), and arterial blood gases parameters collected at baseline during spontaneous ventilation with a conventional face mask and after 1, 12, 24, and 48 hours of initiation of HFNO.

These five variables were used to develop a risk-scoring system to predict HFNO failure. Each data point is assigned such that the sum represented the HACOR score. The HACOR score ranged between 0 to 25 points; higher score suggest an increased risk of HFNO failure [4]. We recorded the duration of HFNO therapy, length ICU and hospital stay and survival. Also, associated complications of HFNO were identified. Patients were followed up until death or hospital discharge.

\section{High flow nasal oxygen settings}

Patients who met inclusion criteria were treated by HFNO. The HFNO device (Optiflow, Fisher \& Paykel Healthcare, Auckland, New Zealand) was applied through a heated humidifier and delivered continuously through large-bore bi-nasal prongs. HFNO was initially administered with a gas flow rate of $50 \mathrm{Lmin}^{-1}$ and an $\mathrm{FiO}_{2}$ of 1.0 and subsequently adjusted to maintain $\mathrm{SpO}_{2}$ of $92 \%$ or more.

The following criteria were used for endotracheal intubation [ETI]:

loss of consciousness; hypotension (e.g, systolic arterial blood pressure $<90 \mathrm{mmHg}$ or mean arterial blood pressure $<65 \mathrm{~mm} \mathrm{Hg}$ ) despite adequate fluid resuscitation, or need for vasopressors;

or two of the following criteria: frank worsening of respiratory distress, $\mathrm{RR}>40$ breaths/min, $\mathrm{SpO}_{2} \leq 92 \%$ despite an $\mathrm{FiO}_{2}$ of 1.0, and/or pH < 7.35. Failure was defined by the need for endotracheal intubation [5].

\section{Statistical analysis}

Data was collected and analyzed using SPSS (Statistical Package for the Social Science, version 20, IBM, and Armonk, New York). Continuous variables were expressed as mean \pm SD while nominal data was expressed in the form of frequency (percentage).

Chi-squared or Fisher's exact tests were used to determine the significance of differences in the observed data. A stepwise multivariate regression analysis performed to assess HFNO failure, and results presented as odds ratio (OR) with 95\% confidence interval (CI). Diagnostic accuracy of 
Table 1. Clinical characteristics of the patients at the enrolment

\begin{tabular}{|c|c|c|c|}
\hline Variables & $\begin{array}{l}\text { Success } \\
(n=100)\end{array}$ & $\begin{array}{c}\text { Failure } \\
(\mathrm{n}=50)\end{array}$ & $\mathbf{P}$ \\
\hline Age [year] & $65.89 \pm 5.67$ & $66.78 \pm 10.54$ & 0.09 \\
\hline Male sex & $80(80 \%)$ & $30(60 \%)$ & 0.009 \\
\hline $\begin{array}{l}\text { Smoking status } \\
\text { Smoker } \\
\text { Ex-smoker } \\
\text { Non-smoker }\end{array}$ & $\begin{array}{l}55(55 \%) \\
25(25 \%) \\
20(20 \%)\end{array}$ & $\begin{array}{c}28(56 \%) \\
16(32 \%) \\
6(12 \%)\end{array}$ & 0.06 \\
\hline $\begin{array}{l}\text { Causes of acute respiratory failure } \\
\text { Community acquired pneumonia } \\
\text { Pulmonary embolism } \\
\text { Cardiogenic pulmonary edema } \\
\text { Acute respiratory distress syndrome }\end{array}$ & $\begin{array}{c}80(80 \%) \\
15(15 \%) \\
2(2 \%) \\
3(3 \%)\end{array}$ & $\begin{array}{c}40(80 \%) \\
5(10 \%) \\
3(6 \%) \\
2(4 \%)\end{array}$ & 0.51 \\
\hline Hypertension [n\%] & $40(40 \%)$ & $15(30 \%)$ & 0.32 \\
\hline Diabetes mellitus [n\%] & $32(32 \%)$ & $18(36 \%)$ & 0.42 \\
\hline C-reactive protein & $26.4 \pm 8.7$ & $28.8 \pm 12.3$ & 0.321 \\
\hline LDH & $442 \pm 321$ & $657 \pm 432$ & 0.001 \\
\hline APACHE-II score & $13.77 \pm 3.68$ & $19.78 \pm 4.09$ & 0.001 \\
\hline HACOR score & $5.56 \pm 2.09$ & $7.50 \pm 1.11$ & 0.001 \\
\hline
\end{tabular}

Data expressed as frequency (percentage), mean (SD). HFNO — high flow nasal oxygen; LDH — lactate dehydrogenase; APACHE-II — acute physiology and chronic health evaluation; HACOR — heart rate, acidosis, consciousness, oxygenation, and respiratory rate

HACOR scale in the prediction of failed HFNO was determined with a ROC curve. Level of confidence was kept at $95 \%$ and hence, the P-value was significant if $<0.05$.

\section{Results}

150 patients with acute hypoxemic respiratory failure, who were receiving HFNO, were enrolled in the study, out of them 100 (66.7\%) subjects had successful HFNO while 50 (33.3\%) patients had failed HFNO. Patient's clinical data were presented in Table 1 . There were no significant differences found in age, sex, and aetiology of respiratory failure at the time of admission. Patients who failed HFNO had significantly higher LDH, APACHE-II and HACOR score.

Changes of physiological parameters between baseline T0 (on the initiation of HFNO), the first hour after enrolment (T1) and after 24 hours (T2) in nasal oxygen success and failure groups are shown in Table 2.

One hour after the enrolment (T1), HR, and $\mathrm{RR}$ improved in success group as compared to failure group ( $111 \pm 16$ vs $120 \pm 20$ beats/minute, $29 \pm 12$ vs $34 \pm 14$ breath/minute), respectively. Improvement was maintained after 24 hours of therapy (T2). There was also improvement in $\mathrm{PaO}_{2} / \mathrm{FiO}_{2}$ after one hour in success group $(185.58 \pm 58.5$ vs $155.07 \pm 52.7)$, which was maintained after $24(201.53 \pm 66.9$ vs $175.6 \pm$ 63.1) hours of therapy. No difference was found in blood pressure and $\mathrm{PaCO}_{2}$.

A summary of HACOR scores at different time point from the initiation of HFNO treatment to $48 \mathrm{~h}$ of HFNO is shown in Table 3 . Patients with HFNO failure had a higher HACOR score at initiation and after 1, 12, 24 and $48 \mathrm{hr}$ than those with success group. Before intubation, the highest value of the HACOR score was reached in patients with HFNO failure.

As presented in Table 4, the predictors for failure of HFNO were HACOR score (odds ratio $=4.44,95 \%$ confidence interval $=3.09-8.07 ; \mathrm{P}$ $<0.001$ ) and APACHE-II score (odds ratio $=1.43$, $95 \%$ confidence interval $=2.01-5.78 ; \mathrm{P}<0.001$ ) with adjusted $\mathrm{R}^{2}$ was 0.65 .

The predictive power of HFNO failure diagnosed by HACOR score is summarized in Table 5. After $1 \mathrm{hr}$ of HFNO assessment, the area under the receiver operating characteristic curve was 0.86 , showing good predictive power for failure. It was noticed that using HACOR score at cut off point $>6$ had $81.2 \%$ sensitivity, $91 \%$ specificity, $92.5 \%$ positive predictive value, and 71.4\% negative predictive value for prediction of HFNO failure with a diagnostic accuracy was $85 \%$. Moreover, the overall diagnostic accuracy exceeded $87 \%$ when the HACOR score was assessed at 1, 12, 24 or $48 \mathrm{hr}$ of HFNO. 
Table 2. Comparisons of physiological parameters between high flow nasal oxygen success and failure groups

\begin{tabular}{|c|c|c|c|}
\hline & $\begin{array}{l}\text { Success } \\
(n=100)\end{array}$ & $\begin{array}{c}\text { Failure } \\
(\mathrm{n}=50)\end{array}$ & $\mathbf{P}$ \\
\hline \multicolumn{4}{|l|}{ SBP [mm Hg] } \\
\hline Baseline (T0) & $120.7 \pm 13.8$ & $120.7 \pm 12.1$ & 0.32 \\
\hline After one hour (T1) & $123.5 \pm 16.5$ & $121.5 \pm 14.5$ & 0.43 \\
\hline After 24 hours (T2) & $124.5 \pm 18.9$ & $123.5 \pm 16.9$ & 0.61 \\
\hline \multicolumn{4}{|l|}{$\mathrm{DBP}[\mathrm{mm} \mathrm{Hg}]$} \\
\hline Baseline (TO) & $72.3 \pm 12.7$ & $70.9 \pm 12.4$ & 0.36 \\
\hline After one hour (T1) & $76.2 \pm 14.5$ & $73.2 \pm 12.8$ & 0.31 \\
\hline After 24 hours (T2) & $76.2 \pm 13.7$ & $76.6 \pm 14.0$ & 0.34 \\
\hline \multicolumn{4}{|l|}{ Heart rate [beat/minute] } \\
\hline Baseline (T0) & $124 \pm 22$ & $123 \pm 23$ & 0.32 \\
\hline After one hour (T1) & $111 \pm 16$ & $120 \pm 20$ & 0.01 \\
\hline After 24 hour (T2) & $98 \pm 13$ & $110 \pm 18$ & 0.01 \\
\hline \multicolumn{4}{|l|}{ RR [breath/minute] } \\
\hline Baseline (TO) & $34 \pm 14$ & $33 \pm 16$ & 0.321 \\
\hline After one hour (T1) & $29 \pm 12$ & $34 \pm 14$ & 0.001 \\
\hline After 24 hours (T2) & $22 \pm 10$ & $30 \pm 13$ & 0.001 \\
\hline \multicolumn{4}{|l|}{$\mathrm{pH}^{+}$} \\
\hline Baseline (TO) & $7.39 \pm 0.11$ & $7.38 \pm 0.10$ & 0.04 \\
\hline After one hour (T1) & $7.40 \pm 0.09$ & $7.38 \pm 0.09$ & $<0.001$ \\
\hline After 24 hour (T2) & $7.43 \pm 0.08$ & $7.39 \pm 0.10$ & 0.01 \\
\hline \multicolumn{4}{|l|}{$\mathrm{PaO}_{2} / \mathrm{FiO}_{2}$} \\
\hline Baseline (TO) & $144.7 \pm 56.8$ & $139.8 \pm 44.5$ & $<0.001$ \\
\hline After one hour (T1) & $185.58 \pm 58.5$ & $155.07 \pm 52.7$ & $<0.001$ \\
\hline After 24 hours (T2) & $201.53 \pm 66.9$ & $175.6 \pm 63.1$ & $<0.001$ \\
\hline \multicolumn{4}{|l|}{$\mathrm{PaCO}_{2}$} \\
\hline Baseline (TO) & $38.3 \pm 13.7$ & $38.9 \pm 14.4$ & 0.51 \\
\hline After one hour (T1) & $39.2 \pm 12.6$ & $38.2 \pm 12.8$ & 0.42 \\
\hline After 24 hours (T2) & $40.0 \pm 9.7$ & $37.6 \pm 13.0$ & 0.21 \\
\hline \multicolumn{4}{|l|}{ GCS } \\
\hline Baseline (TO) & $14.2 \pm 1.4$ & $14.3 \pm 1.2$ & 0.32 \\
\hline After one hour (T1) & $14.4 \pm 1.2$ & $14.5 \pm 1.4$ & 0.23 \\
\hline After 24 hours (T2) & $14.5 \pm 1.2$ & $14.1 \pm 1.1$ & 0.41 \\
\hline
\end{tabular}

Data expressed as mean (SD). P value was significant if $<0.05$. (TO) — at initiation of HFNO; SBP — systolic blood pressure; DBP — diastolic blood pressure; PaCO — arterial carbon dioxide tension; $\mathrm{FiO}_{2}$ — fraction of inspired oxygen; GCS — Glasgow Coma Scale

\section{Table 3. HACOR score at different time point}

\begin{tabular}{lccc}
\hline Time points & $\begin{array}{c}\text { Success } \\
(\mathbf{n}=\mathbf{1 0 0})\end{array}$ & $\begin{array}{c}\text { Failure } \\
(\mathbf{n}=\mathbf{5 0 )}\end{array}$ & P value \\
\hline Initiation of HFNO & $4.8 \pm 2.3$ & $7.4 \pm 3.4$ & 0.001 \\
After 1 hour & $2.5 \pm 2.2$ & $7.5 \pm 3.7$ & 0.001 \\
After 12 hours & $2.0 \pm 2.1$ & $8.3 \pm 4.1$ & 0.001 \\
After 24 hours & $1.6 \pm 1.7$ & $8.1 \pm 4.3$ & 0.001 \\
After 48 hours & $1.3 \pm 1.4$ & $8.4 \pm 4.1$ & 0.001 \\
Intubation & - & $9.5 \pm 4.3$ & - \\
\hline
\end{tabular}

HFNO - high flow nasal oxygen; HACOR — heart rate, acidosis, consciousness, oxygenation, and respiratory rate

\section{Table 4. Predictors of failure of HFNO}

\begin{tabular}{lccc}
\hline Predictors & OR & 95\% Cl & P value \\
\hline APACHE-II & 1.43 & $1.01-3.78$ & $<0.001$ \\
HACOR & 4.44 & $3.09-8.07$ & $<0.001$ \\
$\mathrm{LDH}$ & 1.21 & $1.11-2.03$ & 0.324 \\
C- reactive protein & 1.01 & $0.9-1.9$ & 0.541 \\
\hline \multicolumn{2}{l}{ Variables after 1 hour of HFNO } & & \\
\hline $\mathrm{pH}^{+} \geq 7.35$ & 2.32 & $1.3-3.2$ & 0.432 \\
$\mathrm{PaO}_{2} / \mathrm{FiO}_{2} \geq 200$ & 2.03 & $1.2-3.6$ & 0.02 \\
\hline
\end{tabular}

$\mathrm{OR}$ - odds ratio; $\mathrm{Cl}$ - confidence interval; $\mathrm{LDH}$ - lactate dehydrogenase; APACHE-II — acute physiology and chronic health evaluation; HFNO — high flow nasal oxygen; HACOR — heart rate, acidosis, consciousness, oxygenation, and respiratory rate 
Table 5. Predictive power of HACOR score assessed at 1, 12, 24, and 48 hours in prediction of failed HFNO

\begin{tabular}{lcccc}
\hline Indices & $\mathbf{1 ~ h}$ & $\mathbf{1 2} \mathbf{~ h}$ & $\mathbf{2 4} \mathbf{~}$ & $\mathbf{4 8} \mathbf{~}$ \\
\hline & & Cut off point $>$ 6 & \\
\hline Sensitivity [\%] & 81.2 & 78.2 & 75.4 & 75.2 \\
Specificity [\%] & 91 & 90 & 88 & 88 \\
Positive predictive value [\%] & 92.5 & 90.2 & 91.3 & 91.1 \\
Negative predictive value [\%] & 71.4 & 72.2 & 70.4 & 70.2 \\
Diagnostic accuracy [\%] & 85 & 84 & 85 & 87 \\
AUC [95\% Cl] & 0.86 & 0.84 & 0.82 & 0.83 \\
& $(0.84-0.90)$ & $(0.82-0.90)$ & $(0.80-0.88)$ & $(0.82-0.86)$ \\
\hline
\end{tabular}

$\mathrm{HACOR}$ — heart rate, acidosis, consciousness, oxygenation, and respiratory rate; AUC — area under the curve of receiver operating characteristics; $\mathrm{Cl}$ — confidence interval

Table 6. Mortality rate, length of stay and incidence of complications associated with HFNO

\begin{tabular}{lccc}
\hline & $\begin{array}{c}\text { Success } \\
(\mathbf{n = 1 0 0 )}\end{array}$ & $\begin{array}{c}\text { Failure } \\
\text { (n= 50) }\end{array}$ & P \\
\hline Duration of high-flow nasal oxygen treatment [days] & $4.1 \pm 1.3$ & $3.5 \pm 2.5$ & 0.229 \\
Length of ICU stay [days] & $4.7 \pm 2.22$ & $12.2 \pm 3.30$ & 0.001 \\
Length of hospital stay [days] & $8.2 \pm 3.2$ & $15.1 \pm 5.1$ & 0.001 \\
Mortality at day 28 [n \%] & $3(3 \%)$ & $7(14 \%)$ & 0.01 \\
In-hospital mortality, $\mathrm{n}^{\circ}$ [\%] & $3(3 \%)$ & $12(24 \%)$ & 0.001 \\
\hline Complications associated with HFN0 & & $24(48 \%)$ & 0.001
\end{tabular}

Data expressed as mean (SD), frequency (percentage)

The mean lengths of ICU and hospital stay were significantly higher in HFNO failure in comparison to the success group Table 6. In-hospital mortality rate was higher in HFNO failure patients compared to success group [12 (24\%) vs 3 (3\%); $p=0.008]$ respectively. The only reported complication associated with HFNO was gastric distension in 24 (48\%) failure patients vs $10(10 \%)$ in success groups $(\mathrm{p}=0.001$; Table 5$)$.

\section{Discussion}

HFNO has been gaining traction as an initial treatment in patients with acute hypoxemic respiratory failure. High-flow nasal oxygen was associated with an increased degree of comfort, a reduction in the severity of dyspnea, and a decreased respiratory rate [8]. However, many patients fail HFNO and ultimately need intubation. Early intubation or close observation can benefit patients at risk for HFNO failure [9]. Hence, we evaluate the diagnostic performance of the HACOR score to predict HFNO failure in patients with hypoxemic respiratory failure admitted to a respiratory ICU to avoid delaying intubation and decreased hospital mortality.

This score takes into account heart rate, acidosis, consciousness, oxygenation, and respiratory rate. Because the parameters in the HACOR score are simple bedside measurements, it can serve as a rapid and useful tool for predicting HFNO failure. The current study revealed that $33 \%$ of patients ultimately failed HFNO and predicting the need for intubation. Moreover, we noted that HACOR score at the first hour of initiation and a cutoff point of 6 has a sensitivity of $81 \%$ and a specificity of $91 \%$, with a good diagnostic accuracy $85 \%$.

In agreement with our results, a study conducted by Duan et al. who assessed the usefulness of HACOR scale for prediction of NIV failure in patients with acute hypoxemic respiratory failure. The authors found that HACOR score of $>5 \mathrm{had}$ a higher risk of NIV failure. Thus, recognition of high risk patients and early intubation may presumably reduce hospital mortality [4]. 


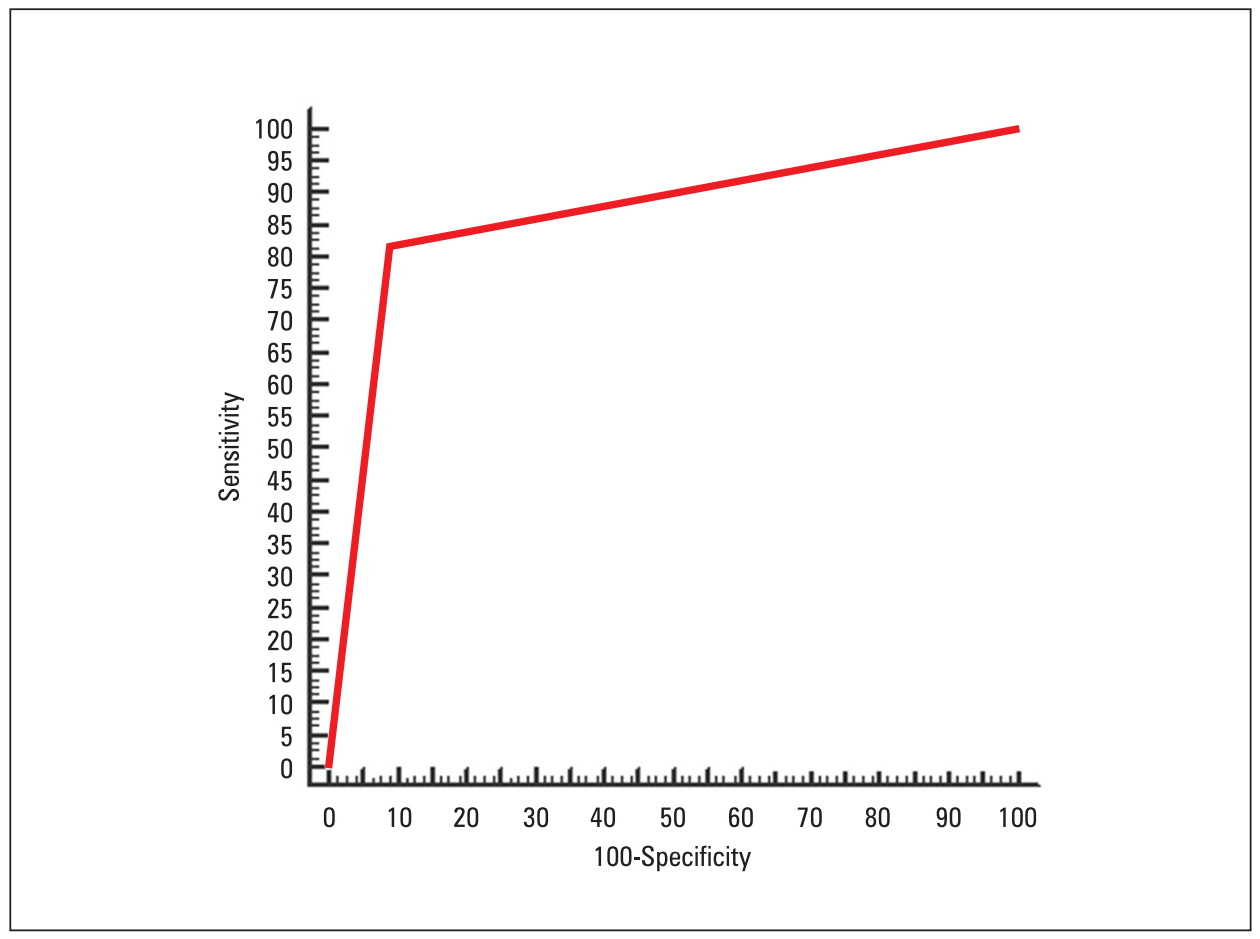

Figure 1. Accuracy of HACOR score in prediction of failed high flow nasal oxygen

Also, Duan et al. studied a novel and practical risk-scoring system to predict noninvasive ventilation (NIV) failure, using bedside clinical variables; 500 chronic obstructive pulmonary disease (COPD) patients were enrolled in a derivation cohort. The authors demonstrated that NIV failure rate was $18.8 \%, 18.9 \%$ and $8.9 \%$ in derivation, internal-validation and external-validation cohorts, respectively. In addition, the HACOR score had good diagnostic power for NIV failure when it was assessed at $1 \mathrm{hr}$ of NIV initiation [10].

The present study, the overall mortality was $24 \%$ in patients with HFNO failure. Therefore, early identification of HFNO failure and intubation is a promising strategy to improve outcome. Recently, in a FLORALI study, a randomized trial consisting of 310 patients with acute hypoxemic respiratory failure allocated to HFNO and standard oxygen therapy. The authors noted that intubation and mortality rate was significantly lower in the HFNO group than in standard oxygen [11].

The points of strength in this study; we assessed the performance of a very useful and a newly developed score in hypoxemic subjects using HFNO. This score has not been previously addressed in High flow nasal oxygen aiming to improve the clinical management and patient's outcome. Limitation in this study, we didn't assess mean flow and $\mathrm{FIO}_{2}$ used by HFNO in the studied population.
Conclusions: HACOR is a newly developed scoring system which takes into account heart rate, acidosis, consciousness, oxygenation, and respiratory rate to predict failure of HFNO in patients with hypoxemic respiratory failure. The score appears to be an effective way of predicting HFNO failure. This could be a promising tool for the clinician to recognize and detect early failure; to ensure that there is no delay in intubation. Patients with a higher HACOR score are more likely to experience failed HFNO. With a cutoff value $<6$, the diagnostic accuracy of the HACOR scale was high. Thus, the HACOR score has been identified as a useful tool to pinpoint patients that will benefit from such intervention.

\section{Ethical approval and consent to participate}

The research received ethical approval from the Ethics Committee of the Faculty of Medicine. The data were confidential. All procedures in the current study were performed according to the ethical standards of the institutional research committee.

\section{Acknowledgements}

We thank all the staffs and patients in ICU who participate in data collection. 


\section{Institutional review board statement}

This study was approved by the Faculty of Medicine Ethics and Scientific Research Committees.

\section{Availability of data and materials}

The datasets used and analyzed during the current study are available from the corresponding author on reasonable request.

\section{Conflict of interest}

None declared.

\section{References:}

1. Roca O, Riera J, Torres F, et al. High-flow oxygen therapy in acute respiratory failure. Respir Care. 2010; 55(4): 408-413, indexed in Pubmed: 20406507.

2. Sztrymf B, Messika J, Mayot T, et al. Impact of high-flow nasal cannula oxygen therapy on intensive care unit patients with acute respiratory failure: a prospective observational study. J Crit Care. 2012; 27(3): 324.e9-324.13, doi: 10.1016/j. jcrc.2011.07.075, indexed in Pubmed: 21958974.

3. Peters SG, Holets SR, Gay PC. High-flow nasal cannula therapy in do-not-intubate patients with hypoxemic respiratory distress. Respir Care. 2013; 58(4): 597-600, doi: 10.4187/respcare.01887, indexed in Pubmed: 22781059.
4. Duan J, Han X, Bai L, et al. Assessment of heart rate, acidosis, consciousness, oxygenation, and respiratory rate to predict noninvasive ventilation failure in hypoxemic patients. Intensive Care Med. 2017; 43(2): 192-199, doi: 10.1007/s00134-0164601-3, indexed in Pubmed: 27812731.

5. Agarwal R, Handa A, Aggarwal AN, et al. Outcomes of noninvasive ventilation in acute hypoxemic respiratory failure in a respiratory intensive care unit in North India. Respir Care. 2009; 54: 1679-87.

6. Brochard L, Mancebo J, Wysocki M, et al. Noninvasive ventilation for acute exacerbations of chronic obstructive pulmonary disease. N Engl J Med. 1995; 333(13): 817-822, doi: 10.1056/ NEJM199509283331301, indexed in Pubmed: 7651472.

7. Knaus WA, Draper EA, Wagner DP, et al. APACHE II: a severity of disease classification system. Crit Care Med. 1985; 13(10): 818-829, indexed in Pubmed: $\underline{3928249}$.

8. Spoletini G, Alotaibi M, Blasi F, et al. Heated humidified highflow nasal oxygen in adults: mechanisms of action and clinical implications. Chest. 2015; 148(1): 253-261, doi: 10.1378/ chest.14-2871, indexed in Pubmed: 25742321.

9. Kang BJu, Koh Y, Lim CM, et al. Failure of high-flow nasal cannula therapy may delay intubation and increase mortality. Intensive Care Med. 2015; 41(4): 623-632, doi: $10.1007 /$ s00134-015-3693-5, indexed in Pubmed: 25691263.

10. Duan J, Bai L, Duan J, et al. Early prediction of noninvasive ventilation failure in COPD patients: derivation, internal validation, and external validation of a simple risk score. Ann Intensive Care. 2019; 9(1): 108, doi: 10.1186/s13613-019-0585-9, indexed in Pubmed: $\underline{31565779}$.

11. Frat JP, Thille A, Mercat A, et al. High-Flow oxygen through nasal cannula in acute hypoxemic respiratory failure. New England Journal of Medicine. 2015; 372(23): 2185-2196, doi: 10.1056/nejmoa1503326. 